\title{
PAPER \\ Fixed-Rate Resource Exchange for Multi-Operator Pico eNodeB
}

\author{
Tomohiko MIMURA $^{\dagger \text { a })}$, Student Member, Koji YAMAMOTO ${ }^{\dagger}$, Senior Member, Masahiro MORIKURA ${ }^{\dagger}$, Fellow, \\ Ayako IWATA ${ }^{\dagger \dagger}$, Member, and Takashi TAMURA ${ }^{\dagger \dagger}$, Nonmember
}

\begin{abstract}
SUMMARY In this paper, we introduce a new multi-operator pico eNodeB (eNB) concept for cellular networks. It is expected that mobile data offloading will be performed effectively after installing the pico eNBs in cellular networks, owing to the rapid increase in mobile traffic. However, when several different operators independently install the pico eNBs, high costs and large amounts of space will be required for the installation. In addition, when several different operators accommodate their own user equipments (UEs) in the pico eNBs, not enough UEs can be accommodated. This is because the UEs are not evenly distributed in the coverage area of the pico eNBs. In this paper, the accommodation of the UEs of different operators in co-sited pico eNB is discussed as one of the solutions to these problems. For the accommodation of the UEs of different operators, wireless resources should be allocated to them. However, when each operator independently controls his wireless resources, the operator is not provided with an incentive to accommodate the UEs of the other operators in his pico eNBs. For this reason, an appropriate rule for appropriate allocation of the wireless resources to the UEs of different operators should be established. In this paper, by using the concepts of game theory and mechanism design, a resource allocation rule where each operator is provided with an incentive to allocate the wireless resources to the UEs of different operators is proposed. With the proposed rule, each operator is not required to disclose the control information like link quality and the number of UEs to the other operators. Furthermore, the results of a throughput performance evaluation confirm that the proposed scheme improves the total throughput as compared with individual resource allocation.

key words: multi-operator, pico eNodeB, resource allocation, game theory, mechanism design
\end{abstract}

\section{Introduction}

Recently, the 3rd Generation Partnership Project (3GPP) standardized the Long Term Evolution-Advanced (LTEAdvanced) as the next-generation mobile communication system. In the LTE-Advanced system, data rates of up to $1 \mathrm{Gbit} / \mathrm{s}$ at $100 \mathrm{MHz}$ bandwidth are supported in the downlink.

For the LTE-Advanced system, a new concept of network sharing has been proposed. This concept is gaining attention because it has the potential to reduce both the infrastructure and environmental costs [1], [2]. Network sharing is a concept of multiple operators sharing the same eNodeB (eNB) or spectrum resources. It was introduced as a topic in the 3GPP Release 10 standard [3]. For example, up

Manuscript received February 13, 2013.

Manuscript revised July 7, 2013.

${ }^{\dagger}$ The authors are with the Graduate School of Informatics, Kyoto University, Kyoto-shi, 606-8501 Japan.

${ }^{\dagger}$ The authors are with the R\&D Division, Panasonic Corporation, Yokohama-shi, 224-8539 Japan.

a)E-mail: info13@imc.cce.i.kyoto-u.ac.jp

DOI: 10.1587/transcom.E96.B.2913 to six operators are allowed to share the same eNB [4], and network sharing can be applied to various communication systems. The effective use of limited resources by network sharing is expected to become a more common agenda [5].

In addition, the installation of small-cell pico eNBs in the coverage area of large-cell macro eNBs for mobile data offloading is gaining more attention. By the installation of the pico eNBs, the area spectral efficiency is expected to be improved [6], [7].

However, when each operator individually installs the pico eNBs and accommodates only his own user equipments (UEs), the number of UEs accommodated in the pico eNBs of each operator is reduced and thus the pico eNBs are not always effectively used. This is because the UEs of each operator are not evenly distributed. Furthermore, there is a high increase in the cost and space for the installation in order to enhance the coverage area of the pico eNBs. Particularly, in an area where the space for the installation of the pico eNBs is limited, such as an underground shopping area and an underground railway, the number of pico eNBs is expected to be reduced.

In this paper, we discuss multi-operator pico eNBs by introducing the concept of network sharing. As shown in Fig. 1, by accommodating the UEs of different operators in the pico eNBs, all the operators can use their pico eNBs more effectively.

We discuss the allocation of the wireless resources to the UEs of different operators to accommodate the UEs of different operators in the pico eNBs. When there is no rule

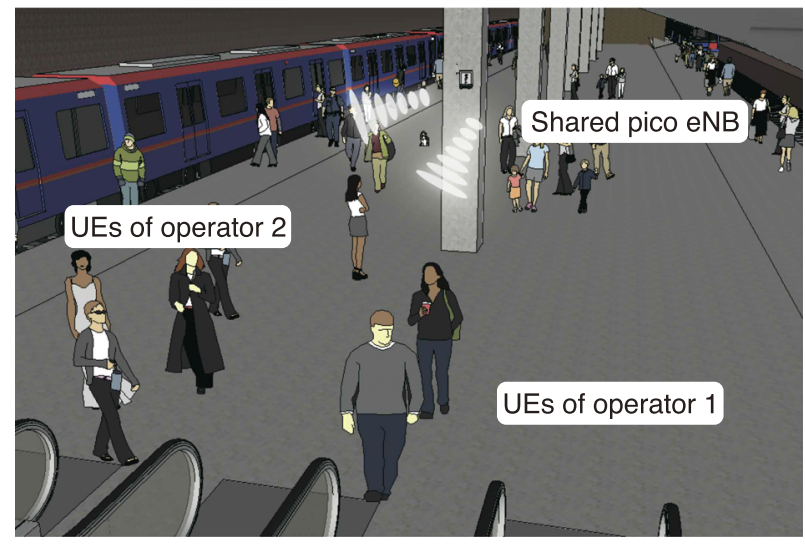

Fig. 1 Multi-operator pico eNB. 
for resource allocation between the operators and when each operator individually controls his resource, each operator is not provided with an incentive to allocate the resources to the UEs of different operators. As a result, the UEs cannot be accommodated in the pico eNBs of different operators and the pico eNBs are not effectively shared.

These problems can be solved by establishing an appropriate rule for resource allocation where each operator is provided with an incentive to accommodate the UEs of different operators. This rule should be informationally decentralized. In other words, it is expected that each operator is not required to disclose the information on the number of UEs or channel quality. In addition, it is expected that the operators will always receive some benefit by joining the system.

In this paper, we propose a fixed-rate resource exchange scheme by introducing the concepts of game theory and mechanism design. Game theory is one of the economic theories which enables the modeling of interactions among multiple rational players. It is applied to the concept of mechanism design. The mechanism design is a theoretical framework to achieve the establishment of rules in a society consisting of rational agents. In addition, it is confirmed that the proposed scheme satisfies the above requirements and improves the total throughput.

The remainder of this paper is organized as follows. In Sect. 2, we describe the concepts of game theory and mechanism design. In Sect. 3, we present the network system model comprising multi-operator pico eNBs and describe the need for establishing a rule for resource allocation between the operators. In Sect. 4, we propose a fixed-rate resource exchange scheme by applying the mechanism design and pure exchange economy model. In Sect. 5, we evaluate the total throughput performance and the amount of exchanged resources by theoretical calculation. In Sect. 6, we present our conclusions.

\section{Game Theory and Mechanism Design}

In this section, the concepts of game theory and mechanism design are described. First, a strategic form game is introduced as the simplest example of game theory. By using the framework of the strategic form game, the interactions among multiple rational players can be discussed. Further, the concept of mechanism design is described. In the mechanism design, the establishment of the rules in the society consisting of multiple rational players can be discussed. Further, some of the research studies on exchange economy as a branch of mechanism design are introduced.

\subsection{Strategic Form Game}

A strategic form game consists of a set of players $\mathcal{I}=$ $\{1, \ldots, N\}$, a set of strategies for each player $\mathcal{X}_{i}$, and the utility of each player $f_{i}$. Therefore, the strategic form game is denoted as $\left(\mathcal{I},\left\{\mathcal{X}_{i}\right\}_{i \in \mathcal{I}},\left\{f_{i}\right\}_{i \in \mathcal{I}}\right)$. Each player chooses a strategy $x_{i} \in \mathcal{X}_{i}$ in order to maximize his utility $f_{i}$. The util- ity $f_{i}$ is dependent on the strategies of the other players $\boldsymbol{x}_{-i}=\left(x_{1}, \ldots, x_{i-1}, x_{i+1}, \ldots, x_{N}\right)$. The best response $x_{i}^{\star}$ is a strategy that maximizes the utility of player $i$ when the strategies of the other players are $\boldsymbol{x}_{-i} . x_{i}^{\star}$ satisfies the following condition:

$$
f_{i}\left(x_{i}^{\star}, \boldsymbol{x}_{-i}\right) \geq f_{i}\left(x_{i}, \boldsymbol{x}_{-i}\right), \forall x_{i} \in \mathcal{X}_{i} .
$$

In addition, the strategy vector $\boldsymbol{x}^{\star}=\left(x_{i}^{\star}, \boldsymbol{x}_{-i}^{\star}\right)$ that every player chooses the best response is called Nash equilibrium. The Nash equilibrium is well known as a solution concept of a strategic form game. At the Nash equilibrium, every player cannot improve his own utility by changing the strategy as follows:

$$
f_{i}\left(x_{i}^{\star}, \boldsymbol{x}_{-i}^{\star}\right) \geq f_{i}\left(x_{i}, \boldsymbol{x}_{-i}^{\star}\right), \forall x_{i} \in \mathcal{X}_{i}, \forall i \in \mathcal{I} .
$$

\subsection{Mechanism Design}

Let a set of players be denoted as $\mathcal{J}=\{1, \ldots, M\}$ and a set of the available resource allocations be denoted as $\mathcal{Y}$. Player $i \in \mathcal{J}$ is assumed to have the preference $\gtrsim_{i}$ on $\mathcal{Y}$. Preference $\gtrsim_{i}$ is the notation of economics and it denotes the order of priority. For example, $\boldsymbol{a} \succsim_{i} \boldsymbol{b}$ means that player $i$ prefers $\boldsymbol{a}$ to $\boldsymbol{b}$. Let the preferences of the players be denoted as $\succsim \equiv\left(\succsim_{1}, \ldots, \succsim_{M}\right)$. When the preferences of the players are $\gtrsim$, a function $g(\gtrsim)$ that chooses the only socially appropriate result from $\mathcal{Y}$ is called a social choice function.

By setting the outcome function $h(\boldsymbol{m})$ from the messages of the players $\boldsymbol{m}=\left(m_{1}, \ldots, m_{M}\right)$, the rule to choose the best distribution can be designed. It can be noted that the message $\boldsymbol{m}$ is transmitted from the players to the manager of the rule and $h(\boldsymbol{m})$ is the function of $\boldsymbol{m}$ and selects the distribution from $\mathcal{X}$. If $\boldsymbol{m}=\gtrsim$, the social objectives can be easily achieved by setting $h=g$. However, the manager does not know the information on the truthful priorities $\gtrsim$, and the joined players have the incentive to transmit strategic massages to the manager in order to maximize their own utility. This results in unfairness among the players and system instability. For this reason, appropriate rules should be established where every player is not required to take strategic actions.

For obtaining an appropriate outcome function, we consider the function $g$ which satisfies the following conditions:

$$
g(\gtrsim) \succsim_{i} g\left(\gtrsim_{i}^{\prime}, \succsim_{-i}\right), \forall i \in \mathcal{J}, \forall \succsim, \forall \gtrsim_{i}^{\prime},
$$

where the false preference of player $i$ is denoted as $\gtrsim_{i}^{\prime}$ and the preferences of the other players are denoted as $\gtrsim_{-i}$. The social choice function $g$ that satisfies this condition satisfies the strategy-proofness. Transmitting genuine messages to the manager is the best response for all the players, regardless of the messages of the other players. In other words, by setting a social choice function $g$ that satisfies the strategyproofness, we need not consider the strategic messages of all the players. As a result, the manager can easily establish the rule and the system instability caused by the strategic 
actions can be overcome.

However, the distribution gained by the social choice function that satisfies the strategy-proofness does not always satisfy the Pareto efficiency and individual rationality. There is a trade-off among the strategy-proofness, Pareto efficiency, and individual rationality.

When there is no distribution $\boldsymbol{b} \in \mathcal{X}$ that satisfies the following conditions:

$$
\begin{aligned}
& \boldsymbol{b} \gtrsim_{i} \boldsymbol{a}, \forall i \in \mathcal{J}, \\
& \boldsymbol{b}>_{i} \boldsymbol{a}, \exists i \in \mathcal{J},
\end{aligned}
$$

the distribution $\boldsymbol{a}$ satisfies Pareto efficiency. It means that every player cannot improve the utility of his own without decreasing the utility of other players.

When the initial distribution $\boldsymbol{w}=\left(w_{1}, \ldots, w_{N}\right)$ satisfies the following condition:

$$
\boldsymbol{a} \succsim_{i} \boldsymbol{w}, \forall i \in \mathcal{J}
$$

the distribution $\boldsymbol{a}$ satisfies the individual rationality. This implies that every player can always improve his own utility by joining the mechanism.

\subsection{Mechanism Design for Exchange Economy}

By using the concept of mechanism design, the resource allocation problems among the operators can be treated as a type of exchange economy. The exchange economy can be modeled as a problem to establish a social choice function $g(\gtrsim)$. There are many research studies on the trade-off among the Pareto efficiency, individual rationality, and strategy-proofness. In 1972, Hurwicz confirmed that there is no social choice function that simultaneously satisfies the Pareto efficiency, individual rationality, and strategy-proofness in two-person and two-goods exchange economies [8]. In 2002, it was confirmed that the Pareto efficiency, individual rationality, and strategy-proofness cannot be simultaneously satisfied in more than two-person and more than two-goods case [9]. In 2003, Serizawa confirmed that there is a serious unfairness in the social choice function that satisfies the Pareto efficiency and strategy-proofness [10]. Barbera and Jackson confirmed that the only exchange rule that satisfies the individual rationality and strategyproofness is the fixed-price trading [11].

\subsection{Strategy-Proof Exchange}

In [11], the exchange economies with a finite number of players who know the information about their preferences are discussed. It is confirmed that the only social choice function that satisfies the strategy-proofness and individual rationality is the fixed-price trading.

The simplest example of the fixed-price trading can be illustrated by using a two-person two-good exchange economy model. Let us assume that player 1 is endowed with $A$ units of good 1 and player 2 is endowed with $B$ units of

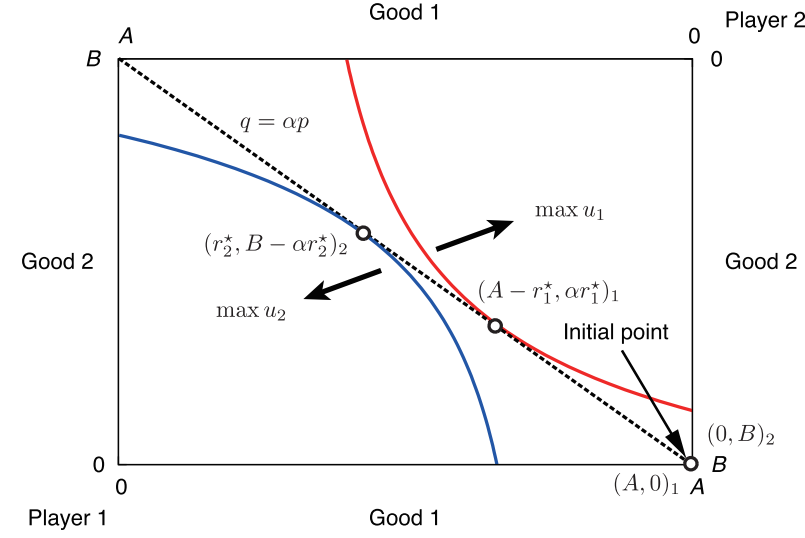

Fig. 2 Fixed-price trading represented by the Edgeworth box.

good 2, as shown in Fig. 2. Each player has a private utility function $u_{i}(p, q)$ depending on the combined units of the amount of goods 1 and 2, where $p$ and $q$ are the number of units of goods 1 and 2, respectively, which are finally allocated to player $i$. The utility functions are continuous and strictly quasi-concave as follows:

$$
u_{i}\left(t p+(1-t) p^{\prime}, t q+(1-q) q^{\prime}\right)>\min \left\{u_{i}(p, q), u_{i}\left(p^{\prime}, q^{\prime}\right)\right\},
$$

$$
\forall(p, q), \forall\left(p^{\prime}, q^{\prime}\right), \forall t,
$$

where $t$ is defined as $0<t<1$ and $(p, q)$ is a point different from $\left(p^{\prime}, q^{\prime}\right)$.

When the pre-specified proportion value is $\alpha$ and the goods are traded between the players corresponding to $\alpha$, each player has only one distribution that maximizes his utility function on $q=\alpha p$. If the point that maximizes the utility function of player 1 is $\left(A-r_{1}^{\star}, \alpha r_{1}^{\star}\right)$, it implies that player 1 is willing to trade $r_{1}^{\star}$ units of good 1 with $\alpha r_{1}^{\star}$ units of good 2 . If the point that maximizes the utility function of player 2 is $\left(r_{2}^{\star}, B-\alpha r_{2}^{\star}\right)$, it implies that player 2 is willing to trade $\alpha r_{2}^{\star}$ units of good 2 with $r_{2}^{\star}$ units of good 1. In this case, by exchanging $\min \left(r_{1}^{\star}, r_{2}^{\star}\right)$ units of $\operatorname{good} 1$ and $\alpha \min \left(r_{1}^{\star}, r_{2}^{\star}\right)$ units of good 2 between the players, the strategy-proofness and individual rationality can be satisfied.

It can be noted that this discussion can be extended to the case of more than two goods, and the fixed-price trading is the only social choice function that satisfies both the strategy-proofness and individual rationality, as proven in [11]. In addition, the fixed-price trading can be extended to the case of more than two-person exchange economies. In this paper, for the sake of simplicity, only the two-person two-good exchange economy model is introduced.

\section{System Model}

In this section, we present the system model for multioperator pico eNBs. Further, we explain the disadvantage of individual resource control and the necessity of the rule to share the radio resources between the operators. 


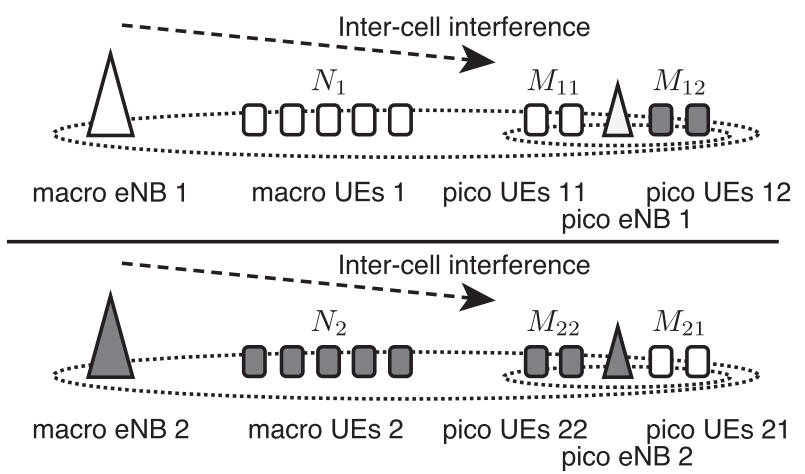

Fig. 3 System model.

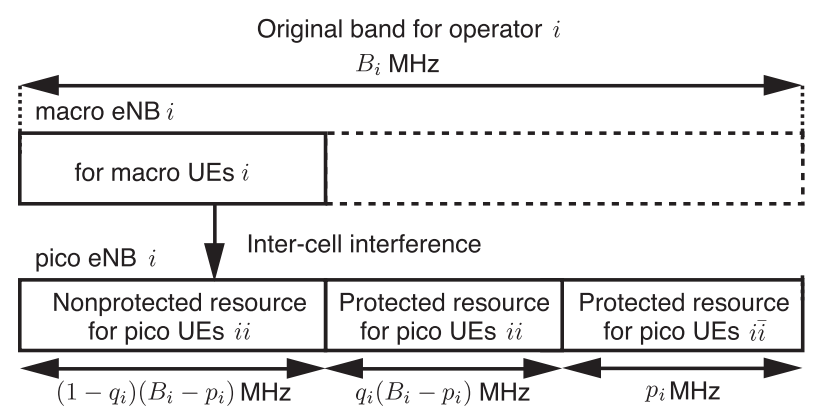

Fig. 4 Original bandwidth of operator $i$.

\subsection{System Model}

In this paper, as the simplest model, we discuss the case where there are two operators. Using the system model, as shown in Fig. 3, operators $i \in\{1,2\}$ individually install macro eNB $i$ and pico eNB $i$. Let the UEs of operator $i$ accommodated in macro eNB $i$ be denoted as macro UEs $i$, and let the UEs of operator $j$ accommodated in pico eNB $i$ be denoted as pico UEs $i j$.

Further, the bandwidth available to each operator is shown in Fig. 4. When one of the operators is operator $i$, let the other operator be denoted as operator $\bar{i}$. Each operator is assigned a fixed amount of dedicated spectrum $B_{i} \mathrm{MHz}$ and allocates $p_{i} \mathrm{MHz}\left(p_{i} \leq B_{i}\right)$ of spectrum to pico UEs $i \bar{i}$.

Furthermore, for the reduction in the interference in the transmitted signal from macro eNB $i$ during communications with pico eNB $i$, an inter-cell interference coordination (ICIC) is introduced [6]. In this paper, for a simple discussion on ICIC, protected and nonprotected resources are introduced [12]. A protected resource is a resource that is used for only pico eNBs-pico UEs communication, and a nonprotected resource is a resource that is used for macro eNBsmacro UEs and pico eNBs-pico UEs communications occurring simultaneously. Operator $i$ can determine the bandwidths of the protected and nonprotected resources. Thus, $q_{i}\left(B_{i}-p_{i}\right)+p_{i} \mathrm{MHz}$ is used as a protected resource and $\left(1-q_{i}\right)\left(B_{i}-p_{i}\right) \mathrm{MHz}$ is used as a nonprotected resource. It can be noted that $q_{i}$ satisfies $0 \leq q_{i} \leq 1$.

\subsection{Individual Resource Control}

The individual resource allocation and ICIC by each operator can be formulated by introducing a strategic form game. When the operators attempt to maximize the product of the user throughputs $u_{i}$ in order to satisfy the fairness of macro UEs and pico UEs, such a resource allocation problem can be formulated as follows:

$$
\begin{aligned}
& \max _{p_{1}, q_{1}} u_{1}\left(p_{1}, p_{2}, q_{1}\right) \\
& =\max _{p_{1}, q_{1}}\left[\frac{r_{\mathrm{m} 1}\left(1-q_{1}\right)\left(B_{1}-p_{1}\right)}{N_{1}}\right]^{N_{1}}\left[\frac{r_{\mathrm{p} 21} p_{2}}{M_{21}}\right]^{M_{21}} \\
& \times\left[\frac{r_{\mathrm{p} 11} q_{1}\left(B_{1}-p_{1}\right)+r_{\mathrm{n} 11}\left(1-q_{1}\right)\left(B_{1}-p_{1}\right)}{M_{11}}\right]^{M_{11}}, \\
& \max _{p_{2}, q_{2}} u_{2}\left(p_{1}, p_{2}, q_{2}\right) \\
& =\max _{p_{2}, q_{2}}\left[\frac{r_{\mathrm{m} 2}\left(1-q_{2}\right)\left(B_{2}-p_{2}\right)}{N_{2}}\right]^{N_{2}}\left[\frac{r_{\mathrm{p} 12} p_{1}}{M_{12}}\right]^{M_{12}} \\
& \times\left[\frac{r_{\mathrm{p} 22} q_{2}\left(B_{2}-p_{2}\right)+r_{\mathrm{n} 22}\left(1-q_{2}\right)\left(B_{2}-p_{2}\right)}{M_{22}}\right]^{M_{22}},
\end{aligned}
$$

where $N_{i}$ is the number of macro UEs $i$ and $M_{i j}$ is the number of pico UEs $i j$. It can be noted that the spectral efficiency of macro eNB $i$-macro UEs $i$ communication is $r_{\mathrm{m} i}$ and the spectral efficiency of a protected (nonprotected) resource for pico eNB $i$-pico UEs $i j$ communication is $r_{\mathrm{p} i j}\left(r_{\mathrm{n} i j}\right)$. Let us assume that $r_{\mathrm{p} i j}>r_{\mathrm{n} i j}$ because there is some interference in a nonprotected resource as compared with a protected resource. Though these maximization problems represent the case where $N_{i} \geq 1$ and $M_{i j} \geq 1$, the resource allocation problems where $N_{i}=0$ or $M_{i j}=0$ can similarly be treated as the maximization problems of $u_{i}$.

Using these conditions,

$$
\begin{aligned}
& u_{1}\left(0, p_{2}, q_{1}\right)>u_{1}\left(p_{1}, p_{2}, q_{1}\right), 0<p_{1} \leq B_{1}, \\
& u_{2}\left(p_{1}, 0, q_{2}\right)>u_{2}\left(p_{1}, p_{2}, q_{2}\right), 0<p_{2} \leq B_{2},
\end{aligned}
$$

are satisfied and strategy $\left(0, q_{i}\right)$ of operator $i$ dominates strategy $\left(p_{i}, q_{i}\right)$. For this reason, $\left(p_{1}^{\star}, p_{2}^{\star}\right)$ is represented as follows:

$$
\left(p_{1}^{\star}, p_{2}^{\star}\right)=(0,0) .
$$

This implies that no resource is allocated to the pico UEs that are accommodated in the pico eNBs of the other operator because each operator is not provided with an incentive to allocate some of the resources to the pico UEs of different operators when they try to maximize the product of the user throughputs. For this reason, a rule where each operator is provided with an incentive to allocate the radio resources to the pico UEs of the other operator should be established.

\section{Fixed-Rate Resource Exchange and Its Property}

In this section, a fixed-rate resource exchange scheme for 
multi-operator pico eNBs is presented as the proposed scheme. We confirm that the proposed scheme satisfies the strategy-proofness and individual rationality. In addition, the total throughput performance is confirmed by theoretical calculation.

\subsection{Fixed-Rate Resource Exchange}

From the viewpoint of the system stability and operator fairness, a fixed-rate resource exchange scheme is proposed as a scheme that satisfies strategy-proofness and individual rationality. In this scheme, we introduce a manager that is independent to both operators, and the manager establishes the allocation rule as shown in Fig. 5. The manager adjusts the amount of resource exchange between the operators.

In the proposed scheme, both the operators report the desired bandwidth $m_{i} \mathrm{MHz}$ to be exchanged. The manager reports $m^{\star}=\min \left(m_{1}, m_{2}\right)$ to the operators. Therefore, operator 1 allocates $m^{\star} \mathrm{MHz}$ and operator 2 allocates $\alpha m^{\star} \mathrm{MHz}$ to the UEs of the other operator. It can be noted that $\alpha$ represents the rate of resource exchange, which is predetermined by the manager.

As shown in Fig. 6, the fixed-rate resource exchange scheme is illustrated by using an Edgeworth box. In this figure, point $\mathrm{O}$ represents the initial resource allocation. The manager decides on the amount of exchanged resource to improve the utility of each operator $u_{i}$ on the line $p_{2}=\alpha p_{1}$. When both the operators report $m_{i}$ to the manager, they cal-

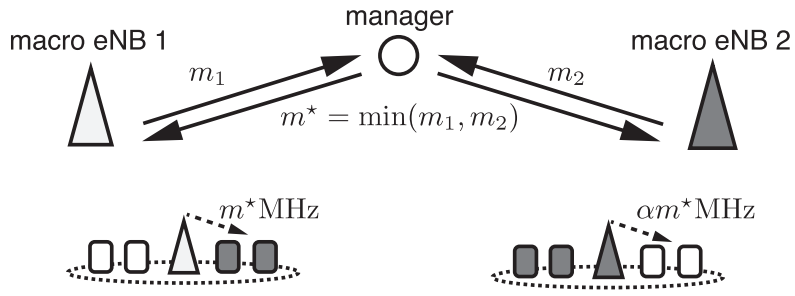

pico UEs 11 pico UEs 12 pico UEs 22 pico UEs 21 pico eNB $1 \quad$ pico eNB 2

Fig. 5 Fixed rate resource exchange.

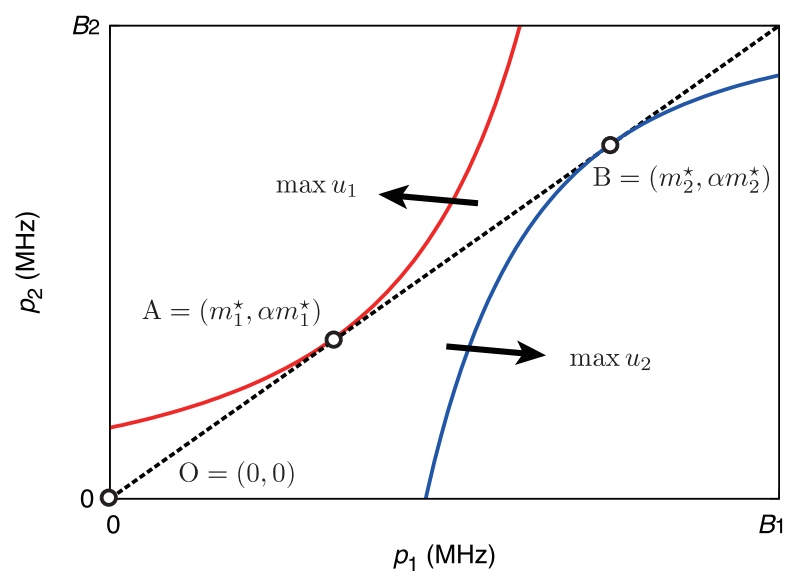

Fig. 6 Fixed rate resource exchange represented by the Edgeworth box. culate the optimal value of $m_{i}$ to maximize their utilities. However, the optimal amount of resource exchange differs for each operator. The best response for operator 1 is point $\mathrm{A}$ and that for operator 2 is point $\mathrm{B}$.

\subsection{Messages of Both Operators}

Let us denote the optimal amount of exchanged resource and the value of $q_{i}$ for operator $i$ as $m_{i}^{\star}$ and $q_{i}^{\star}$, respectively. When $N_{i}$ and $M_{i j}$ are greater than 0 , we can calculate $m_{i}^{\star}$ and $q_{i}^{\star}$ as follows:

$$
\begin{aligned}
& \left(m_{1}^{\star}, q_{1}^{\star}\right)=\underset{m, q_{1}}{\arg \max } u_{1}\left(m, \alpha m, q_{1}\right) \\
& =\underset{m, q_{1}}{\arg \max }\left[\frac{r_{\mathrm{m} 1}\left(1-q_{1}\right)\left(B_{1}-m\right)}{N_{1}}\right]^{N_{1}}\left[\frac{r_{\mathrm{p} 21} \alpha m}{M_{21}}\right]^{M_{21}} \\
& \times\left[\frac{r_{\mathrm{p} 11} q_{1}\left(B_{1}-m\right)+r_{\mathrm{n} 11}\left(1-q_{1}\right)\left(B_{1}-m\right)}{M_{11}}\right]^{M_{11}} \\
& =\underset{m, q_{1}}{\arg \max } \frac{\left[\left(B_{1}-m\right)^{N_{1}+M_{11}} m^{M_{21}}\right] r_{\mathrm{m} 1}{ }^{N_{1}}\left(r_{\mathrm{p} 21} \alpha\right)^{M_{21}}}{N_{1}^{N_{1}} M_{11} M_{11} M_{21}{ }^{M_{21}}} \\
& \times\left(1-q_{1}\right)^{N_{1}}\left[\left(r_{\mathrm{p} 11}-r_{\mathrm{n} 11}\right) q_{1}+r_{\mathrm{n} 11}\right]^{M_{11}}, \\
& \left(m_{2}^{\star}, q_{2}^{\star}\right)=\underset{m, q_{2}}{\arg \max } u_{2}\left(m, \alpha m, q_{2}\right) \\
& =\underset{m, q_{2}}{\arg \max }\left[\frac{r_{\mathrm{m} 2}\left(1-q_{2}\right)\left(B_{2}-\alpha m\right)}{N_{2}}\right]^{N_{2}}\left[\frac{r_{\mathrm{p} 12} m}{M_{12}}\right]^{M_{12}} \\
& \times\left[\frac{r_{\mathrm{p} 22} q_{2}\left(B_{2}-\alpha m\right)+r_{\mathrm{n} 22}\left(1-q_{2}\right)\left(B_{2}-\alpha m\right)}{M_{22}}\right]^{M_{22}} \\
& =\underset{m, q_{2}}{\arg \max } \frac{\left[\left(B_{2}-\alpha m\right)^{N_{2}+M_{22}} m^{M_{12}}\right] r_{\mathrm{m} 2}{ }^{N_{2}} r_{\mathrm{p} 12}{ }^{M_{12}}}{N_{2}{ }^{N_{2}} M_{22}{ }^{M_{22}} M_{12}{ }^{M_{12}}} \\
& \times\left(1-q_{2}\right)^{N_{2}}\left[\left(r_{\mathrm{p} 22}-r_{\mathrm{n} 22}\right) q_{2}+r_{\mathrm{n} 22}\right]^{M_{22}} .
\end{aligned}
$$

In order that operator 1 evaluates (14), operator 1 needs being informed the information on the number of pico UEs $21, M_{21}$, and the spectral efficiency of a protected resource for pico eNB 2-pico UEs 21 communication, $r_{\mathrm{p} 21}$, from operator 2 and vice versa. It means that the proposed fixed rate resource exchange scheme requires additional information compared to the individual resource control. In addition, additional functions between these networks are required so that additional information can be exchanged. Note that even in the proposed scheme, the number of UEs and the spectral efficiency of UEs of the other operator are not required.

By solving these optimization problems, we obtain $m_{i}^{\star}$ and $q_{i}^{\star}$ as follows:

$$
\begin{aligned}
m_{1}^{\star} & =\frac{M_{21} B_{1}}{N_{1}+M_{11}+M_{21}}, \\
m_{2}^{\star} & =\frac{M_{12} B_{2}}{\alpha\left(N_{2}+M_{12}+M_{22}\right)}, \\
q_{1}^{\star} & =1-\frac{N_{1} r_{\mathrm{p} 11}}{\left(r_{\mathrm{p} 11}-r_{\mathrm{n} 11}\right)\left(N_{1}+M_{11}\right)},
\end{aligned}
$$




$$
q_{2}^{\star}=1-\frac{N_{2} r_{\mathrm{p} 22}}{\left(r_{\mathrm{p} 22}-r_{\mathrm{n} 22}\right)\left(N_{2}+M_{22}\right)} .
$$

Of course, $m_{i}^{\star}$ and $q_{i}^{\star}$ can be calculated by solving the optimization problem in the case $N_{i}=0$ or $M_{i j}=0$. When $M_{\overline{i i}}=0$, we obtain

$$
\begin{aligned}
m_{i}^{\star} & =0, \\
q_{i}^{\star} & =1-\frac{N_{i} r_{\mathrm{p} i i}}{\left(r_{\mathrm{p} i i}-r_{\mathrm{n} i i}\right)\left(N_{i}+M_{i i}\right)}, N_{i}+M_{i i} \neq 0,
\end{aligned}
$$

where $q_{i}^{\star}$ is not defined when $N_{i}+M_{i i}=0$. This is because there is no optimization problem for operator $i$ to solve when $N_{i}+M_{i i}+M_{\overline{i i}}=0$.

When $M_{\overline{i i}} \neq 0$ and $N_{i}+M_{i i}=0$, we obtain

$$
m_{1}^{\star}=B_{1}, m_{2}^{\star}=\frac{B_{2}}{\alpha},
$$

where $q_{i}^{\star}$ is not defined. This is because operator $i$ has no macro UEs $i$ or pico UEs $i j$ to allocate his radio resource. In the other cases, $m_{i}^{\star}$ and $q_{i}^{\star}$ can be represented as (16)-(19).

It can be noted that $m_{i}^{\star}$ is not the amount of exchanged resource, which is finally determined by the manager. Operator $i$ can maximize utility $u_{i}$ only when the manager finally determines the amount of exchanged resource $m^{\star}=m_{i}^{\star}$. However, $m_{1}^{\star}$ and $m_{2}^{\star}$ are different values. For this reason, both the utilities $u_{1}$ and $u_{2}$ are not always maximized by the decision of the manager.

$q_{i}^{\star}$ is constant regardless of $m^{\star}$. This implies that each operator can set his optimal $q_{i}^{\star}$ before the manager sets the amount of exchanged resource $m^{\star}$. In the following, $q_{i}$ is assumed to be set as $q_{i}^{\star}$. Let us denote $u_{i}\left(m, \alpha m, q_{i}^{\star}\right)$ as $v_{i}(m)$ because $q_{i}^{\star}$ can be treated as a constant value.

Theorem 1: $\quad v_{i}(m)$ has only one local maximum at $m=m_{i}^{\star}$.

Proof 1: By the definition of $v_{i}(m), v_{1}(m)$ and $v_{2}(m)$ are represented as follows:

$$
\begin{aligned}
& v_{1}(m)=C_{1}\left(B_{1}-m\right)^{N_{1}+M_{11}} m^{M_{21}}, \\
& v_{2}(m)=C_{2}\left(B_{2}-\alpha m\right)^{N_{2}+M_{22}} m^{M_{12}},
\end{aligned}
$$

where $C_{i}$ is a positive constant value, $M_{\overline{i i}} \neq 0$, and $N_{i}+N_{i i} \neq$ 0 .

$\mathrm{d} v_{1}(m) / \mathrm{d} m$ and $\mathrm{d} v_{2}(m) / \mathrm{d} m$ are calculated as follows:

$$
\begin{gathered}
\frac{\mathrm{d} v_{1}(m)}{\mathrm{d} m}=C_{1}\left[M_{21} B_{1}-m\left(N_{1}+M_{11}+M_{21}\right)\right] \\
\times\left(B_{1}-m\right)^{N_{1}+M_{11}-1} m^{M_{21}-1} \\
\frac{\mathrm{d} v_{2}(m)}{\mathrm{d} m}=C_{2}\left[M_{12} B_{2}-\alpha m\left(N_{2}+M_{22}+M_{12}\right)\right] \\
\times\left(B_{2}-\alpha m\right)^{N_{2}+M_{22}-1} m^{M_{12}-1} .
\end{gathered}
$$

It is confirmed that $\mathrm{d} v_{1}(m) / \mathrm{d} m=0$ when $m \in\left\{0, m_{1}^{\star}, B_{1}\right\}$ and $\mathrm{d} v_{2}(m) / \mathrm{d} m=0$ when $m \in\left\{0, m_{2}^{\star}, B_{2} / \alpha\right\}$. Furthermore, $\mathrm{d} v_{1}(m) / \mathrm{d} m>0$ when $0<m<m_{1}^{\star}$ and $\mathrm{d} v_{1}(m) / \mathrm{d} m<0$ when $m_{1}^{\star}<m<B_{1}$ and $B_{1}<m$. Similarly, $\mathrm{d} v_{2}(m) / \mathrm{d} m>0$ when $0<m<m_{2}^{\star}$ and $\mathrm{d} v_{2}(m) / \mathrm{d} m<0$ when $m_{2}^{\star}<m<B_{2} / \alpha$ and $B_{2} / \alpha<m$. As a result, $v_{i}(m)$ has the only one local maximum at $m=m_{i}^{\star}$. follows:

If $M_{\bar{i} i} \neq 0$ and $N_{i}+M_{i i}=0, \mathrm{~d} v_{i}(m) / \mathrm{d} m$ is calculated as

$$
\frac{\mathrm{d} v_{i}(m)}{\mathrm{d} m}=C_{i} M_{\overline{i i}} m^{M_{i i}-1} .
$$

It is confirmed that $\mathrm{d} v_{i}(m) / \mathrm{d} m>0$ if $m \neq 0$. As a result, $v_{i}(m)$ has the only one local maximum at $m=m^{\star}$.

If $M_{\bar{i} i}=0$ and $N_{i}+M_{i i} \neq 0, \mathrm{~d} v_{1}(m) / \mathrm{d} m$ and $\mathrm{d} v_{2}(m) / \mathrm{d} m$ are calculated as follows:

$$
\begin{aligned}
& \frac{\mathrm{d} v_{1}(m)}{\mathrm{d} m}=-C_{1}\left(N_{1}+M_{11}\right)\left(B_{1}-m\right)^{N_{1}+M_{11}-1}, \\
& \frac{\mathrm{d} v_{2}(m)}{\mathrm{d} m}=-C_{2} \alpha\left(N_{2}+M_{22}\right)\left(B_{2}-\alpha m\right)^{N_{2}+M_{22}-1} .
\end{aligned}
$$

It is confirmed that $\mathrm{d} v_{1}(m) / \mathrm{d} m<0$ if $B_{1} \neq m$ and $\mathrm{d} v_{2}(m) / \mathrm{d} m<0$ if $B_{2} \neq \alpha m$. As a result, $v_{i}(m)$ has only one local maximum at $m=m^{\star}=0$.

For the abovementioned reasons, it is confirmed that $v_{i}(m)$ has only one local maximum at $m=m_{i}^{\star}$.

This implies that $v_{i}(m)$ is improved when the final amount of exchanged resource $m^{\star}$ is close to $m_{i}^{\star}$. When the amount of exchanged resource is too small, the throughput performance of the UEs accommodated in the pico eNBs of the other operator is degraded. On the contrary, when the amount of exchanged resource is too large, the macro UEs and pico UEs of the same operator cannot achieve enough throughput.

However, $m_{i}^{\star}$ is unknown to the manager, and each operator is provided with an incentive to report a strategic message $m_{i} \neq m_{i}^{\star}$ to maximize his own utility.

\subsection{Strategy-Proofness and Individual Rationality}

In this section, we confirm that the proposed scheme satisfies both the strategy-proofness and individual rationality.

Theorem 2: The proposed scheme satisfies the strategyproofness.

Proof 2: As summarized in Table 1, the utility of operator 1 versus the messages of each operator $\left(m_{1}, m_{2}\right)$ is shown. Let us denote the strategic message $m_{1}$ that is lower than $m_{1}^{\star}$ as $L$ and that higher than $m_{1}^{\star}$ as $H$. As summarized in Table 1, the pairs of messages are divided into 12 patterns.

When $m_{2} \leq L$, operator 1 can achieve a utility of $v_{1}\left(m_{2}\right)$ regardless of his message $m_{1}$. This is because the manager determines $m^{\star}$ by the calculation of $\min \left(m_{1}, m_{2}\right)$.

When $L \leq m_{2} \leq m_{1}^{\star}$, operator 1 can achieve a utility

Table 1 The utility of operator 1 when the messages of both operators are $\left(m_{1}, m_{2}\right)$.

\begin{tabular}{|c|c|c|c|c|}
\hline & $m_{2} \leq L$ & $L \leq m_{2} \leq m_{1}^{\star}$ & $m_{1}^{\star} \leq m_{2} \leq H$ & $H \leq m_{2}$ \\
\hline$L=m_{1} \leq m_{1}^{\star}$ & $v_{1}\left(m_{2}\right)$ & $v_{1}(L)$ & $v_{1}(L)$ & $v_{1}(L)$ \\
\hline$m_{1}=m_{1}^{\star}$ & $v_{1}\left(m_{2}\right)$ & $v_{1}\left(m_{2}\right)$ & $v_{1}\left(m_{1}^{\star}\right)$ & $v_{1}\left(m_{1}^{\star}\right)$ \\
\hline$m_{1}^{\star} \leq m_{1}=H$ & $v_{1}\left(m_{2}\right)$ & $v_{1}\left(m_{2}\right)$ & $v_{1}\left(m_{2}\right)$ & $v_{1}(H)$ \\
\hline
\end{tabular}


of $v_{i}(L)$ when he reports $m_{1}=L$. However, he can achieve $v_{i}\left(m_{2}\right)$ when he reports $m_{1}=m_{1}^{\star}$ or $m_{1}^{\star}=H$. It is confirmed that $m_{1}=m_{1}^{\star}$ or $m_{1}^{\star}=H$ is the best response for operator 1 when $L \leq m_{2} \leq m_{1}^{\star}$. This is because $v_{1}(m)$ has only one local maximum when $m=m_{1}^{\star}$ and $v_{1}(L) \leq v_{m_{2}}$.

When $m_{1}^{\star} \leq m_{2} \leq H$ or $H \leq m_{2}, m_{1}=m_{1}^{\star}$ is the best response with which the utility of $v_{1}\left(m_{1}^{\star}\right)$ can be achieved, and it is the maximum value of $v_{1}(m)$.

Overall, the reporting message $m_{1}=m_{1}^{\star}$ is the best response for operator 1 regardless of $m_{2}$. Similarly, the reporting message $m_{2}=m_{2}^{\star}$ is the best response for operator 2 . Thus, the proposed scheme satisfies the strategy-proofness.

Theorem 3: The proposed scheme satisfies the individual rationality.

Proof 3: Operator 1 can achieve the utility of $v_{1}\left(m_{2}\right)$ when $m_{2} \leq m_{1}^{\star}$ and $v_{1}\left(m_{1}^{\star}\right)$ when $m_{1}^{\star} \leq m_{2}$. This is because the best response of operator 1 is the reporting message $m_{1}=$ $m_{1}^{\star}$.

In the case where $0 \leq m_{2} \leq m_{1}^{\star}$, we obtain $v_{1}(0) \leq$ $v_{1}\left(m_{2}\right)$ because $v_{i}(m)$ has only one local maximum when $m=m_{1}^{\star}$. In the case where $m_{1}^{\star} \leq m_{2} \leq 1$, we obtain $v_{1}(0) \leq v_{1}\left(m_{1}^{\star}\right)$. In other words, the utility of operator 1 , with the proposed scheme, is always greater than that of the individual resource allocation $v_{1}(0)$. Similarly, the utility of operator 2 is also greater than $v_{2}(0)$.

Thus, the proposed scheme satisfies the individual rationality and both operators 1 and 2 always improve their utilities.

\subsection{Theoretical Performance}

The amount of exchanged resource $m^{\star}$ can be determined as follows:

$$
p_{1}=m^{\star}, p_{2}=\alpha m^{\star}, m^{\star}=\min \left(m_{1}^{\star}, m_{2}^{\star}\right) .
$$

When $m_{1}^{\star} \geq m_{2}^{\star}$ as shown in Fig. $6, \mathrm{~A}=\left(m_{1}^{\star}, \alpha m^{\star}\right)$ is the final allocation result.

In addition, we determine the total throughput performance of operator $i, t_{i}$, as follows:

$$
\begin{aligned}
t_{1}=r_{\mathrm{p} 11} q_{1}^{\star} & \left(B_{1}-m^{\star}\right)+\alpha r_{\mathrm{p} 21} m^{\star} \\
& +\left(r_{\mathrm{m} 1}+r_{\mathrm{n} 11}\right)\left(1-q_{1}^{\star}\right)\left(B_{1}-m^{\star}\right), \\
t_{2}=r_{\mathrm{p} 22} q_{2}^{\star} & \left(B_{2}-\alpha m^{\star}\right)+r_{\mathrm{p} 12} m^{\star} \\
+ & \left(r_{\mathrm{m} 2}+r_{\mathrm{n} 22}\right)\left(1-q_{2}^{\star}\right)\left(B_{2}-\alpha m^{\star}\right) .
\end{aligned}
$$

\section{Numerical Results}

\subsection{Parameters}

In this section, the total throughput and the amount of exchanged resource are evaluated by using the examples of the parameters as listed in Table 2 . The average spectral efficiency in each link is set to a fixed value. Note the effect
Table 2 Example of parameters.

\begin{tabular}{c|c}
\hline$\left(N_{1}, N_{2}\right)$ & $(5,5),(20,20)$ \\
$r_{\mathrm{m} 1}, r_{\mathrm{m} 2}$ & $2 \mathrm{bit} / \mathrm{s} / \mathrm{Hz}$ \\
$r_{\mathrm{p} 11}, r_{\mathrm{p} 12}, r_{\mathrm{p} 21}, r_{\mathrm{p} 22}$ & $5 \mathrm{bit} / \mathrm{s} / \mathrm{Hz}$ \\
$r_{\mathrm{n} 11}, r_{\mathrm{n} 22}$ & $0.5 \mathrm{bit} / \mathrm{s} / \mathrm{Hz}$ \\
$B_{1}, B_{2}$ & $20 \mathrm{MHz}, 20 \mathrm{MHz}$ \\
\hline
\end{tabular}

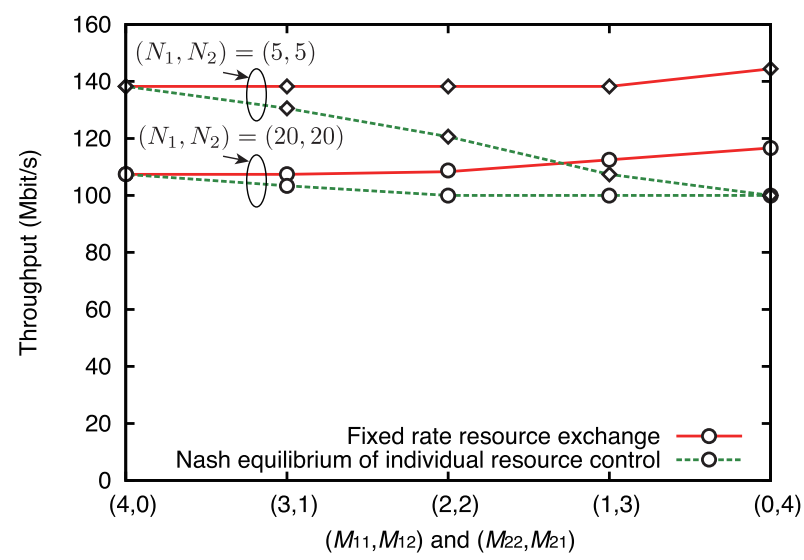

Fig. 7 Total throughput performance of both operators against the number of UEs accommodating in pico eNBs.

of the fading is averaged out. The spectrum efficiency of the macro eNB-macro UEs communication is set to $2 \mathrm{bit} / \mathrm{s} / \mathrm{Hz}$. The spectrum efficiency of the protected resource for the pico eNB-pico UEs communication is set to $5 \mathrm{bit} / \mathrm{s} / \mathrm{Hz}$, and that of the nonprotected resource is set to $0.5 \mathrm{bit} / \mathrm{s} / \mathrm{Hz}$. The number of UEs accommodated in the macro eNBs and pico eNBs varies. The amount of dedicated spectrum for each operator $B_{i}$ is set to $20 \mathrm{MHz}$, and the rate of resource exchange $\alpha$ is set to 1 .

\subsection{Total Throughput}

As shown in Fig. 7, the total throughput performances of the proposed scheme and individual resource control are compared. We obtain the total throughput performance of individual resource control, $T$, by the calculation of the Nash equilibrium as follows:

$$
T=\sum_{i=1}^{2}\left[\left(r_{\mathrm{m} i}+r_{\mathrm{n} i i}\right)\left(1-q_{i}^{\star}\right)+r_{\mathrm{p} i i} q_{i}^{\star}\right] B_{i} .
$$

As shown in Fig. 7, as $M_{12}$ and $M_{21}$ increase, the total throughput of the individual resource control decreases. This is because both the operators are not provided with an incentive to allocate the resources to the pico UEs of the other operator. This implies that the pico UEs are not accommodated in the pico eNBs of the other operator when there is no rule for resource allocation between the operators, and both the operators attempt to maximize their utilities without considering the other operator.

With the proposed scheme, the total throughput performance is improved. When $\left(M_{11}, M_{12}\right)$ and $\left(M_{22}, M_{21}\right)$ are set to $(2,2)$, the total throughput is improved by from 10 to 


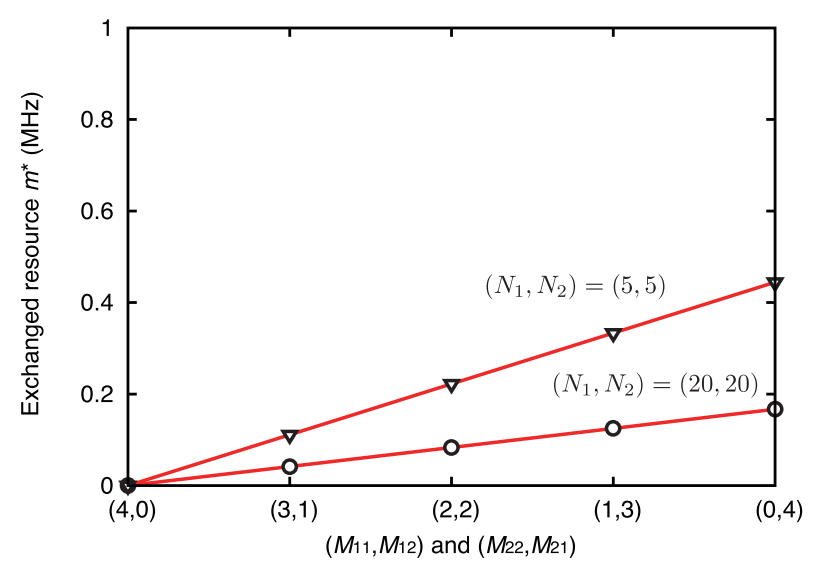

Fig. 8 The amount of exchanged resource by the proposed scheme.

Table 3 Example of parameters in the unbalanced situation.

\begin{tabular}{c|c|c}
\hline & Case 1 & Case 2 \\
\hline$\left(N_{1}, N_{2}\right)$ & $(5,5),(20,20)$ & $(5,20)$ \\
$r_{\mathrm{m} 1}, r_{\mathrm{m} 2}$ & $2 \mathrm{bit} / \mathrm{s} / \mathrm{Hz}$ & $2 \mathrm{bit} / \mathrm{s} / \mathrm{Hz}$ \\
$r_{\mathrm{p} 11}, r_{\mathrm{p} 12}, r_{\mathrm{p} 21}, r_{\mathrm{p} 22}$ & $5 \mathrm{bit} / \mathrm{s} / \mathrm{Hz}$ & $5 \mathrm{bit} / \mathrm{s} / \mathrm{Hz}$ \\
$r_{\mathrm{n} 11}, r_{\mathrm{n} 22}$ & $0.5 \mathrm{bit} / \mathrm{s} / \mathrm{Hz}$ & $0.5 \mathrm{bit} / \mathrm{s} / \mathrm{Hz}$ \\
$B_{1}, B_{2}$ & $20 \mathrm{MHz}, 10 \mathrm{MHz}$ & $20 \mathrm{MHz}, 20 \mathrm{MHz}$ \\
\hline
\end{tabular}

$20 \%$ compared with the individual resource control. Particularly, when $\left(M_{11}, M_{12}\right)$ and $\left(M_{22}, M_{21}\right)$ are set to $(0,4)$, the total throughput is improved by from 20 to $40 \%$. This is because more protected resources are allocated to pico UEs 12 and pico UEs 21 by using the proposed scheme.

\subsection{Amount of Exchanged Resource}

The amount of exchanged resource, with the proposed scheme, $m^{\star}$, is shown in Fig. 8. It is confirmed that a large amount of resource is exchanged when $M_{12}$ and $M_{21}$ are large, regardless of $N_{1}$ and $N_{2}$. This is because the both operators simultaneously require a large amount of resource for pico UEs 12 and pico UEs 21.

Furthermore, when $N_{1}$ and $N_{2}$ are small, it is confirmed that a large amount of resource is exchanged. This is because there are a few macro UEs and both the operators require to allocate a large amount of resource to their pico UEs in order to maximize the product of the user throughputs. On the contrary, when $N_{1}$ and $N_{2}$ are large, both the operators require to allocate a large amount of resource to the macro UEs. Therefore, the amount of exchanged resource is small.

\subsection{Unbalanced Situations}

In sections from 5.1 to 5.3, the system performance is evaluated when the number of UEs, the amount of required resources, and the bandwidth of operators are balanced. To confirm the throughput improvement of the proposed scheme in unbalanced situations, we conduct additional evaluations using parameters summarized in Table 3 . In case $1, B_{1}$ and $B_{2}$ are different from each other and the other parameters are the same as Table 2. In case $2,\left(N_{1}, N_{2}\right)$ is set

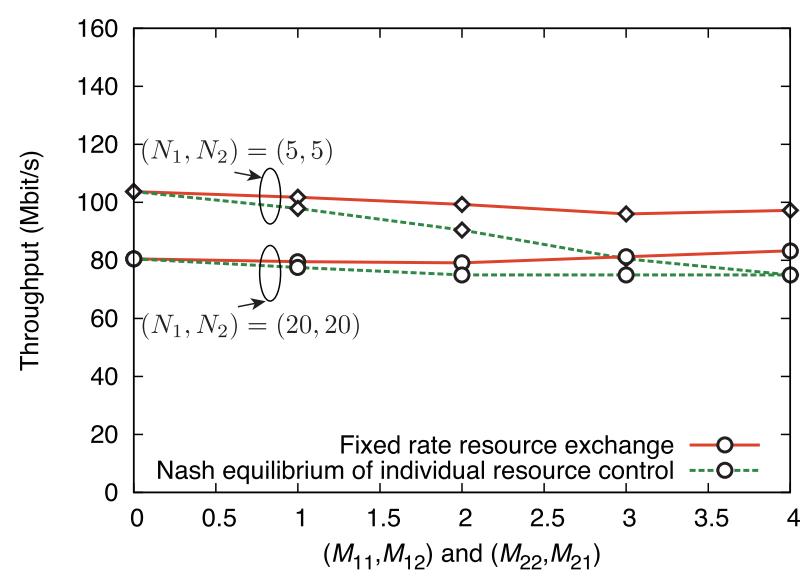

Fig. 9 Total throughput performance of both operators against the number of UEs accommodating in pico eNBs in case 1.

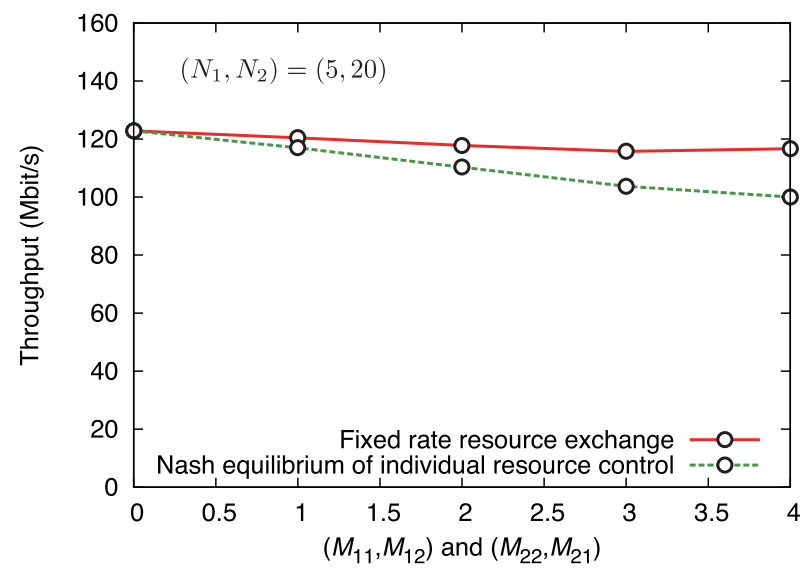

Fig. 10 Total throughput performance of both operators against the number of UEs accommodating in pico eNBs in case 2 .

to $(5,20)$ and the other parameters are the same.

Figure 9 shows the total throughput in case 1, i.e., $B_{1}$ and $B_{2}$ are different from each other. Figure 10 shows the total throughput in case 2, i.e., $N_{1}$ and $N_{2}$ are different from each other. In both cases, we confirmed that the total throughput performance of the proposed scheme is improved compared with that of individual resource control. However, the amount of throughput improvement in unbalanced situations is smaller than that in balanced situations because the amount of exchanged resource is determined by the smaller value of desired bandwidths from two operators.

\section{Conclusion}

In this paper, accommodation of the UEs of different operators in the pico eNBs is discussed. When the UEs of different operators are accommodated in the pico eNBs, the wireless resources for the communication are not appropriately allocated to them. This is because the operator who owns the pico eNBs is not provided with an incentive to allocate the wireless resources to the UEs of the other operators.

To solve this problem, we introduce the concepts of 
game theory and mechanism design. The resource allocation problem for multi-operator pico eNBs is discussed by using the pure exchange economy model. In this paper, a fixed-rate resource exchange scheme is proposed to satisfy the strategy-proofness and individual rationality in the resource allocation.

It is confirmed from the theoretical performance evaluation results that the total throughput performance can be improved when both the operators allocate the wireless resources to maximize the product of the user throughput performances. Furthermore, when the number of UEs in the macro eNBs is small or the number of UEs in different pico eNBs is large, large amounts of resources are exchanged between the operators. This implies that both operators can exchange the wireless resources when they simultaneously require the resource for the UEs accommodated in the pico eNBs of the other operator.

We would like to emphasize that the objective of this paper is to propose a fixed-rate resource exchange scheme for multi-operator pico eNBs and to confirm the effect of the proposed scheme. We hope that the results presented in this paper will provide insights that are useful for the design of heterogeneous network systems.

\section{References}

[1] J. Village, K. Worrall, and D. Crawford, "3G shared infrastructure," Proc. Third International Conference on 3G Mobile Communication Technologies 2002, pp.10-16, London, UK., May 2002.

[2] K. Johansson, M. Kristensson, and U. Schwarz, "Radio resource management in roaming based multi-operator WCDMA networks," Proc. IEEE Vehicular Technology Conference (VTC 2004-Spring), vol.4, pp.2062-2066, Milano, Italy, May 2004.

[3] 3GPP TR22.951, "Service aspects and requirements for network sharing (Release 10)," V10.0.0, March 2011.

[4] 3GPP TS36.300, "E-UTRAN: Overall description (Release 10)," V10.5.0, Oct. 2011.

[5] S. AlQahtani, A. Mahmoud, and A. Sheikh, "Performance analysis of adaptive rate scheduling scheme for 3G WCDMA wireless networks with multi-operators," Proc. IEEE International Conference on Communications (ICC 2007), pp.4811-4816, Glasgow, Scotland, June 2007.

[6] A. Khandekar, N. Bhushan, J. Tinghang, and V. Vanghi, "LTEAdvanced: Heterogeneous networks," Proc. European Wireless Conference 2010, pp.972-982, Lucca, Italy, April 2010.

[7] G. Fodor, C. Koutsimanis, A. Racz, N. Reider, A. Simonsson, and W. Mulleret, "Intercell interference coordination in OFDMA networks and in the 3GPP long term evolution system," Academy Publisher Journal of Commun., pp.445-453, Aug. 2009.

[8] L. Hurwicz, On Informationally Decentralized Systems, Cambridge University Press, 1972.

[9] S. Serizawa, "Inefficiency of stratagy-proof rules for pure exchange economies," J. Economic Theory, vol.106, pp.219-241, Sept. 2002.

[10] S. Serizawa and J. Weymark, "Efficient strategy-proof exchange and minimum consumption guarantees," J. Economic Theory, vol.109, pp.246-263, Sept. 2003.

[11] S. Barbera and M. Jackson, "Strategy-proof exchange," Econometrica, vol.69, pp.51-87, Sept. 1995.

[12] M.R. Jeong and N. Miki, "A simple scheduling restriction scheme for interference coordinated networks," Proc. Vehicular Technology Conference (VTC2012-Fall), pp.1-6, Québec City, Canada, Sept. 2012.

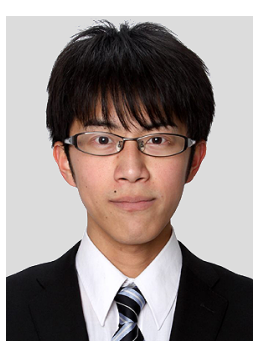

Tomohiko Mimura received the B.E. degree in electrical and electronic engineering from Kyoto University in 2011. He is currently studying toward his M.E. degree at the Graduate School of Informatics, Kyoto University. His research interests include next-generation wireless communication systems and applications of game theory. $\mathrm{He}$ is a student member of the IEEE.

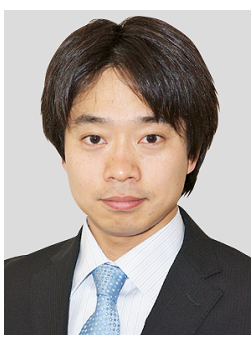

Koji Yamamoto received the B.E. degree in electrical and electronic engineering from Kyoto University in 2002, and the M.E. and Ph.D. degrees in informatics from Kyoto University in 2004 and 2005, respectively. From 2004 to 2005 , he was a research fellow of the Japan Society for the Promotion of Science (JSPS). Since 2005, he has been with the Graduate School of Informatics, Kyoto University, where he is currently an associate professor. From 2008 to 2009 , he was a visiting researcher at Wireless@KTH, Royal Institute of Technology (KTH) in Sweden. His research interests include game theory, spectrum sharing, and M2M networks. He received the PIMRC 2004 Best Student Paper Award in 2004, the Ericsson Young Scientist Award in 2006, and the Young Researcher's Award from the IEICE of Japan in 2008. He is a member of the IEEE.

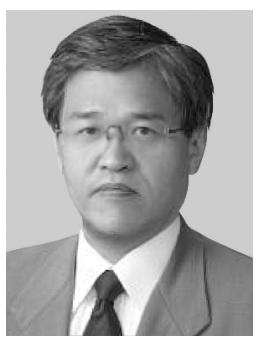

Masahiro Morikura received his B.E., M.E., and Ph.D. degrees in electronics engineering from Kyoto University, Kyoto, Japan in 1979, 1981 and 1991, respectively. He joined NTT in 1981, where he was engaged in the research and development of TDMA equipment for satellite communications. From 1988 to 1989, he was with the Communications Research Centre, Canada, as a guest scientist. From 1997 to 2002, he was active in the standardization of the IEEE 802.11a based wireless

LAN. He received the Paper Award and the Achievement Award from IEICE in 2000 and 2006, respectively. He also received the Education, Culture, Sports, Science and Technology Minister Award in 2007 and Maejima Award in 2008. Dr. Morikura is now a professor in the Graduate School of Informatics, Kyoto University. He is a member of the IEEE.

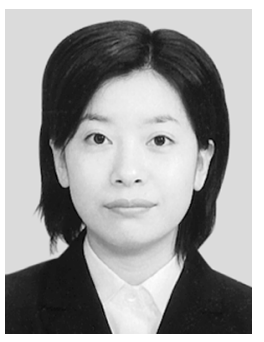

Ayako Iwata received B.E. and M.E. degree from Keio University, Japan in 2001 and 2003, respectively. She joined Panasonic Mobile Communications Co., Ltd., Japan in 2003. She has been with Matsushita Electric Industrial Co., Ltd. and Panasonic Corporation, Japan since 2004. She is currently engaged in the research and development of mobile communication system and standardization. She was a recipient of the IEICE RCS (Radio Communication System) Active Research Award, 2009. 


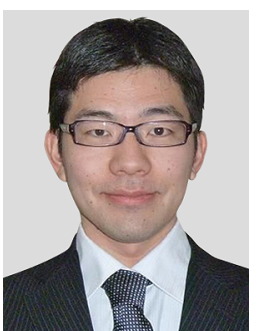

Takashi Tamura received B.E. and M.E. degree from Osaka University, Japan in 2005 and 2007, respectively. He joined Matsushita Electric Industrial Co., Ltd., Japan in 2007. $\mathrm{He}$ has been with Panasonic Corporation, Japan since 2008. He was engaged in the research and development of mobile communication systems and standardization. 